\title{
Quantum Statistical Relation for black holes in nonlinear electrodynamics coupled to Einstein-Gauss-Bonnet AdS gravity
}

\author{
Olivera Miskovid* and Rodrigo Oleat \\ Instituto de Física, \\ Pontificia Universidad Católica de Valparaíso, \\ Casilla 4059, Valparaíso, Chile
}

(Dated: October 16, 2018)

\begin{abstract}
We consider curvature-squared corrections to Einstein-Hilbert gravity action in the form of Gauss-Bonnet term in $D>4$ dimensions. In this theory, we study the thermodynamics of charged static black holes with anti-de Sitter (AdS) asymptotics, and whose electric field is described by nonlinear electrodynamics (NED). These objects have received considerable attention in recent literature on gravity/gauge dualities.

It is well-known that, within the framework of anti de-Sitter/Conformal Field Theory (AdS/CFT) correspondence, there exists a nonvanishing Casimir contribution to the internal energy of the system, manifested as the vacuum energy for global AdS spacetime in odd dimensions. Because of this reason, we derive a Quantum Statistical Relation directly from the Euclidean action and not from the integration of the First Law of thermodynamics. To this end, we employ a background-independent regularization scheme which consists in the addition to the bulk action of counterterms that depend on both extrinsic and intrinsic curvatures of the boundary (Kounterterm series). This procedure results in a consistent inclusion of the vacuum energy and chemical potential in the thermodynamic description for Einstein-Gauss-Bonnet AdS gravity regardless the explicit form of the NED Lagrangian.

PACS numbers: 04.50.-h, 04.50.Gh, 04.70.Dy, 11.30.-j
\end{abstract}

\section{INTRODUCTION}

AdS/CFT correspondence [1] provides a computational tool to obtain physical properties of a field theory at strong coupling by studying its gravity dual.

In hydrodynamic models, for example, gauge/gravity duality is used to calculate the ratio of shear viscosity $\eta$ to entropy density $s$ in a strongly interacting quantum theory. In particular, it predicts a universal bound $1 / 4 \pi$ of this ratio for a large class of theories, dual to Einstein AdS gravity [2].

However, it has been shown that the addition of higher-derivative terms to the gravitational action in AdS space modifies the dynamics of the boundary theory and it violates the KSS bound [3]. On the other hand, higher-curvature terms in Abelian fields described by nonlinear electrodynamics (NED) do not change the value $\eta / s[4]$, unless gravity action is augmented by a Gauss-Bonnet (GB) term, what also causes instabilities in the large-momentum regime [5].

*Electronic address: olivera.miskovic@ucv.cl

${ }^{\dagger}$ Electronic address: rodrigo.olea@ucv.cl 
In a similar fashion, when studying gravity duals to high critical temperature superconductivity models, the inclusion of GB and NED terms alters the ratio between $T_{c}$ and the energy gap [6 8 ].

In the examples of AdS/CFT duality mentioned above, the mapping between the bulk and boundary theories makes an extensive use of thermal properties of black holes in the gravity side of the correspondence. This provides the motivation to investigate the thermodynamics of black holes in Einstein-Gauss-Bonnet (EGB) gravity with negative cosmological constant coupled to an arbitrary NED theory. In particular, we prove here that any black hole which is a solution to the theory will satisfy the Quantum Statistical Relation (QSR) [10]

$$
T I^{E}=U-T S+\Phi Q
$$

where $T$ is the Hawking temperature, $U$ is the black hole energy and $S$ its entropy that includes a correction due to GB term. The addition of a NED Lagrangian to pure gravity action brings in electric charge $Q$ to the solution, whose conjugate variable $\Phi$ is the difference in electric potential between the horizon and infinity. In other words, the Euclidean action is the Legendre transformation of the entropy with respect to chemical (electric) potential. As it is standard within the framework of AdS/CFT, the Euclidean action has to be regularized by means of a backgroundindependent procedure.

In gravity with AdS asymptotics, there is a subtle difference between the relation (11) and the First Law of thermodynamics

$$
d U=T d S-\Phi d Q
$$

because $U$ contains in general a Casimir contribution in $D=2 n+1$ dimensions [11]. Clearly, the latter formula remains the same if the total energy is shifted by an additive constant $E_{v a c}$. This does not mean that the presence of a vacuum energy is irrelevant. On the contrary, it is expected that $E_{v a c}$ will appear in a consistent thermodynamic description of asymptotically AdS (AAdS) black holes, when we work out their thermal properties from the partition function in semiclassical approximation

$$
Z=e^{-I_{\text {clas }}^{E}}
$$

Here, $I_{\text {clas }}^{E}$ stands for the classical Euclidean action, which is divergent in AdS gravity. If one intends to identify any thermodynamic potential with the Euclidean action, one must resort to a regularization mechanism that eliminates the divergences in the infrared sector of the theory, but not the vacuum energy. It is only in this way that we can extract $I^{E}$ as the finite part of $I_{\text {clas }}^{E}$, and relate it to the asymptotic charges that appear in Eq.(1).

In the context of AdS/CFT correspondence, the regularization of the Euclidean action requires the addition of counterterms which are intrinsic functionals of the boundary metric and curvature [12, 13], and constructed using holographic techniques for AAdS spacetimes [14, 15]. When a GB term is included in the gravity action -despite the fact the field equations are still second-order in the metric- one faces almost unsurmountable obstacles to apply the holographic renormalization procedure. Thus, it is necessary to postulate the explicit form of the local counterterm series and, 
then, the coefficients in the series are adjusted by the convergence of charges and Euclidean action for particular solutions [16, 17]. This seems to work well for low enough dimensions, but it is far from giving a general prescription for all cases.

In this paper, we apply an extrinsic, background-independent regularization method for EGB AdS gravity to render the Euclidean action finite in all dimensions [18]. This scheme involves the addition to the action of counterterms depending on both the intrinsic and extrinsic curvatures (which, for that reason, have been suggestively called Kounterterms). This procedure results in the obtention of a Quantum Statistical Relation for black hole solutions to Einstein-Gauss-Bonnet AdS gravity coupled to an arbitrary NED. As we shall show below, the picture in odd dimensions is consistent only if the total energy is shifted as $U=M+E_{v a c}$ with respect to the Hamiltonian mass $M$, in a similar fashion as for Einstein-Born-Infeld case studied in Ref. [19].

\section{FIELD EQUATIONS}

Nonlinear actions for electromagnetic field have been a subject of research for many years, since it was noted by Heisenberg and Euler that quantum corrections to electrodynamics lead to nonlinear equations for the field strength [20]. The physics of objects described by nonlinear effective Lagrangians, as the one of Born and Infeld [21], possesses remarkable properties, such as a regular electric field at the origin and a finite self-energy. Furthermore, Born-Infeld electrodynamics can be obtained in exact one-loop computations from open Bose strings [22].

Gravity coupled to NED leads to a large class of charged black hole solutions. In EinsteinHilbert gravity coupled to Born-Infeld electrodynamics, static, spherically symmetric black holes were derived in, e.g., Refs. [23, 24]. Among other gravitating NED models that exhibit electrically charged solutions, we can mention the logarithmic theory investigated in [25], and the conformally invariant ED found in [26].

We will study gravitating NED in any spacetime dimension $D>4$ which is described by the action

$$
I_{0}=\int_{\mathcal{M}} d^{D} x \sqrt{-g} \mathcal{L}_{0}=I_{\text {grav }}+I_{N E D}
$$

The first part of the bulk action corresponds to the one of Einstein-Hilbert (EH) with negative cosmological constant $\Lambda=-(D-1)(D-2) / 2 \ell^{2}$ and a higher-curvature correction given by the Gauss-Bonnet term

$$
I_{\text {grav }}=\frac{1}{16 \pi G} \int_{\mathcal{M}} d^{D} x \sqrt{-g}\left[R-2 \Lambda+\alpha\left(R^{2}-4 R_{\mu \nu} R^{\mu \nu}+R_{\mu \nu \lambda \sigma} R^{\mu \nu \lambda \sigma}\right)\right] .
$$

Here, $\ell$ is the AdS radius, $G$ is the gravitational constant and $\alpha$ is the GB coupling, which has to be positive if one regards this action as obtained in the low-energy limit of String Theory.

The Abelian gauge field $A_{\mu}(x)$ defines a field strength $F_{\mu \nu}=\partial_{\mu} A_{\nu}-\partial_{\nu} A_{\mu}$, which couples minimally to gravity through the quadratic invariant $F^{2}=g^{\mu \lambda} g^{\nu \rho} F_{\mu \nu} F_{\lambda \rho}$. We consider an arbitrary 
Lagrangian density $\mathcal{L}\left(F^{2}\right)$, such that the NED action has the form

$$
I_{N E D}=\int_{\mathcal{M}} d^{D} x \sqrt{-g} \mathcal{L}\left(F^{2}\right) .
$$

The equations of motion follow from the variation of the bulk action respect to the dynamic fields $g_{\mu \nu}$ and $A_{\mu}$, that is, $\delta I_{0} / \delta g_{\mu \nu}=0$ and $\delta I_{0} / \delta A_{\mu}=0$,

$$
\begin{aligned}
\mathcal{E}_{\nu}^{\mu} & \equiv G_{\nu}^{\mu}+H_{\nu}^{\mu}-8 \pi G T_{\nu}^{\mu}=0 \\
\mathcal{E}^{\mu} & \equiv \nabla_{\nu}\left(F^{\mu \nu} \frac{d \mathcal{L}}{d F^{2}}\right)=0
\end{aligned}
$$

In Eq.(7), $G_{\nu}^{\mu}$ is the Einstein tensor which includes the contribution from the cosmological constant

$$
G_{\nu}^{\mu}=R_{\nu}^{\mu}-\frac{1}{2} \delta_{\nu}^{\mu} R+\Lambda \delta_{\nu}^{\mu}
$$

whereas $H_{\nu}^{\mu}$ denotes the Lanczos tensor

$$
\begin{aligned}
H_{\nu}^{\mu}= & -\frac{\alpha}{2} \delta_{\nu}^{\mu}\left(R^{2}-4 R^{\alpha \beta} R_{\alpha \beta}+R^{\alpha \beta \lambda \sigma} R_{\alpha \beta \lambda \sigma}\right) \\
& +2 \alpha\left(R R_{\nu}^{\mu}-2 R^{\mu \lambda} R_{\lambda \nu}-2 R_{\lambda \nu \sigma}^{\mu} R^{\lambda \sigma}+R^{\mu \alpha \sigma \sigma} R_{\nu \alpha \lambda \sigma}\right),
\end{aligned}
$$

which comes from the GB part of the gravity action. The right hand side of Eq.(17) corresponds to the matter stress tensor $T^{\mu \nu}=\frac{2}{\sqrt{-g}} \frac{\delta I_{N E D}}{\delta g_{\mu \nu}}$ which has the form

$$
T_{\nu}^{\mu}=\delta_{\nu}^{\mu} \mathcal{L}-4 \frac{d \mathcal{L}}{d F^{2}} F^{\mu \lambda} F_{\nu \lambda}
$$

For a given solution ansatz, the generalized Maxwell equation (8) determines the dynamics of the electromagnetic field.

The presence of the higher-curvature term $H_{\nu}^{\mu}$ in the equation of motion (7) changes the vacua structure of AdS gravity. Indeed, a nonvanishing GB coupling $\alpha$ modifies the AdS radius $\ell$ of maximally symmetric spaces to an effective value $\ell_{\text {eff }}$ given by

$$
\ell_{e f f}^{( \pm) 2}=\frac{2 \alpha(D-3)(D-4)}{1 \pm \sqrt{1-\frac{4 \alpha}{\ell^{2}}(D-3)(D-4)}}, \quad \alpha \leq \frac{\ell^{2}}{4(D-3)(D-4)} .
$$

A multiplicity of solutions may exist in either branch of the theory, some of them yet to be discovered. For the present paper we will consider spacetimes which are AAdS. In order to impose this condition in a way independent of any coordinate frame, we will assume the AAdS behavior in the curvature rather than in the metric, that is,

$$
R_{\mu \nu}^{\alpha \beta} \rightarrow-\frac{1}{\ell_{e f f}^{2}} \delta_{[\mu \nu]}^{[\alpha \beta]},
$$

for the corresponding branch.

For static black holes with constant-curvature transversal section $\Gamma_{D-2}$, described by the metric $\gamma_{n m}$, the line element in the local coordinates $x^{\mu}=\left(t, r, \varphi^{m}\right)$ reads

$$
d s^{2}=g_{\mu \nu}(x) d x^{\mu} d x^{\nu}=-f^{2}(r) d t^{2}+\frac{d r^{2}}{f^{2}(r)}+r^{2} \gamma_{m n}(\varphi) d \varphi^{m} d \varphi^{n}
$$


The curvature of $\Gamma_{D-2}$ is labeled by the topological parameter $k=0,+1$ or -1 for flat, spherical or hyperbolic case, respectively. The spacetime boundary $\partial \mathcal{M}$ has the topology of a cylinder and is placed at $r \rightarrow \infty$. A necessary condition for a solution to be a black hole is the existence of a horizon $r_{+}$such that $f^{2}\left(r_{+}\right)=0$. In case the latter relation possesses more than one root, $r_{+}$ denotes the outermost one.

A charged static solution is given in terms of a gauge field which depends only on the radial coordinate

$$
A_{\mu}=\phi(r) \delta_{\mu}^{t}
$$

what generates the electric field

$$
E(r)=-\phi^{\prime}(r)
$$

such that the field strength is

$$
F_{\mu \nu}=E(r)\left(\delta_{\mu}^{t} \delta_{\nu}^{r}-\delta_{\nu}^{t} \delta_{\mu}^{r}\right)
$$

Here, the prime stands for radial derivative.

In the static ansatz (14 17), the gravitational equation (7) gives rise to a single independent differential equation for $f^{2}$,

$$
\begin{aligned}
\mathcal{E}_{t}^{t}= & \mathcal{E}_{r}^{r}=\frac{D-2}{2 r^{2}}\left[r\left(f^{2}\right)^{\prime}+(D-3)\left(f^{2}-k\right)-(D-1) \frac{r^{2}}{\ell^{2}}\right] \\
& +\alpha(D-2)(D-3)(D-4) \frac{k-f^{2}}{r^{3}}\left[\left(f^{2}\right)^{\prime}-(D-5) \frac{k-f^{2}}{2 r}\right]-8 \pi G T_{r}^{r}
\end{aligned}
$$

where

$$
T_{t}^{t}=T_{r}^{r}=\left.\left(\mathcal{L}+4 E^{2} \frac{d \mathcal{L}}{d F^{2}}\right)\right|_{F^{2}=-2 E^{2}} .
$$

On the other hand, the form of the electric field (17) leads to the differential version of a generalized Gauss' law

$$
\mathcal{E}^{t}=-\frac{d}{d r}\left(\left.r^{D-2} E \frac{d \mathcal{L}}{d F^{2}}\right|_{F^{2}=-2 E^{2}}\right)=0,
$$

whose solution introduces $q$ as an integration constant related to the electric charge,

$$
\left.E \frac{d \mathcal{L}}{d F^{2}}\right|_{F^{2}=-2 E^{2}}=-\frac{q}{r^{D-2}}
$$

The electric field decouples from the metric and one can determine $E$ just from this algebraic equation. Therefore, the electric potential at the distance $r$ measured with respect to radial infinity is calculated from

$$
\phi(r)=-\int_{\infty}^{r} d v E(v)
$$


For the analysis of thermodynamic properties of charged black holes, the conjugate variable to the electric charge $Q$ is the difference in electric potential between infinity and the event horizon $r_{+}$, that is, $\Phi=\phi(\infty)-\phi\left(r_{+}\right)$.

The first integral of the equation of motion for the metric (18) is

$$
\left(f^{2}-k\right)\left(1-\alpha(D-3)(D-4) \frac{f^{2}-k}{r^{2}}\right)=\frac{r^{2}}{\ell^{2}}-\frac{\mu}{r^{D-3}}+\frac{16 \pi G \mathcal{T}(q, r)}{(D-2) r^{D-3}},
$$

where $\mu$ is related to the black hole mass, whereas the electromagnetic flux through the surface $r=$ const is given by the function

$$
\begin{aligned}
\mathcal{T}(q, r) & =\int_{\infty}^{r} d v v^{D-2} T_{r}^{r} \\
& =\int_{\infty}^{r} d v\left(v^{D-2} \mathcal{L}-4 q E\right),
\end{aligned}
$$

for an arbitrary NED Lagrangian. In the second line, the charge $q$ appears due to the Gauss law (21).

As mentioned above, the theory possesses two branches, what is manifested as two solutions in the metric function $f^{2}$ of Eq.(23) for EGB gravity coupled to NED,

$$
f_{ \pm}^{2}(r)=k+\frac{r^{2}}{2 \alpha(D-3)(D-4)}\left[1 \pm \sqrt{1-4 \alpha(D-3)(D-4)\left(\frac{1}{\ell^{2}}-\frac{\mu}{r^{D-1}}+\frac{16 \pi G \mathcal{T}(q, r)}{(D-2) r^{D-1}}\right)}\right]
$$

The branch with positive sign $f_{+}^{2}(r)$ has an ill-defined limit $\alpha \rightarrow 0$, and it does not recover the solutions of EH AdS gravity. Furthermore, perturbations around the corresponding vacuum

state are proved to have negative mass [27]. Henceforth, we will focus on the stable and ghost-free branch $f^{2}(r) \equiv f_{-}^{2}(r)$. Asymptotically, this branch and its radial derivative behave as

$$
\begin{aligned}
f^{2} & =k+\frac{r^{2}}{\ell_{e f f}^{2}}-\frac{\mu}{1-\frac{2 \alpha}{\ell_{e f f}^{2}}(D-3)(D-4)} \frac{1}{r^{D-3}}+\mathcal{O}\left(\frac{1}{r^{2 D-6}}\right), \\
\left(f^{2}\right)^{\prime} & =\frac{2 r}{\ell_{e f f}^{2}}+\frac{(D-3) \mu}{1-\frac{2 \alpha}{\ell_{e f f}^{2}}(D-3)(D-4)} \frac{1}{r^{D-2}}+\mathcal{O}\left(\frac{1}{r^{2 D-5}}\right) .
\end{aligned}
$$

The fact that there are no additional contributions to the energy of charged black holes in any physically sensible NED theory is a consequence of the metric fall-off, which is the same as in Reissner-Nordstrom case.

The procedure outlined above can be regarded as an algorithm to construct explicit solutions to various NED theories: conformally invariant electrodynamics, Born-Infeld, Logarithmic electrodynamics, etc., as it was pointed out in Ref. [28].

\section{IYER-WALD CHARGES AND BLACK HOLE ENTROPY}

At this point, we proceed to evaluate the Euclidean continuation of the action for EGB gravity coupled to NED in Eqs.(5) and (6). 
As a first step, without loss of generality, we take Gauss-normal coordinates to foliate the spacetime along a radial direction

$$
d s^{2}=g_{\mu \nu} d x^{\mu} d x^{\nu}=N^{2}(r) d r^{2}+h_{i j}(r, x) d x^{i} d x^{j} .
$$

This frame is obtained as a particular gauge-fixing $\left(N^{i}=0\right)$ of a general ADM form of the metric. We assume the manifold to have a boundary $\partial \mathcal{M}$, located at radial infinity, endowed with an induced metric $h_{i j}$.

The identities for the totally antisymmetric product of Kronecker deltas listed in Appendix A enable us to write down the pure gravity part of the bulk action as

$$
\begin{aligned}
I_{\text {grav }}=\frac{1}{16 \pi G(D-2) !} \int_{\mathcal{M}} d^{D} x \sqrt{-g} \delta_{\left[\nu_{1} \cdots \nu_{D}\right]}^{\left[\mu_{1} \cdots \mu_{D}\right]}\left(\frac{1}{2} R_{\mu_{1} \mu_{2}}^{\nu_{1} \nu_{2}} \delta_{\mu_{3}}^{\nu_{3}} \delta_{\mu_{4}}^{\nu_{4}}\right. \\
\left.\quad+\frac{D-2}{D \ell^{2}} \delta_{\mu_{1}}^{\nu_{1}} \delta_{\mu_{2}}^{\nu_{2}} \delta_{\mu_{3}}^{\nu_{3}} \delta_{\mu_{4}}^{\nu_{4}}+\frac{\alpha(D-2)(D-3)}{4} R_{\mu_{1} \mu_{2}}^{\nu_{1} \nu_{2}} R_{\mu_{3} \mu_{4}}^{\nu_{3} \nu_{4}}\right) \delta_{\mu_{5}}^{\nu_{5}} \cdots \delta_{\mu_{D}}^{\nu_{D}} .
\end{aligned}
$$

In the foliation (28), the indices split as $\mu=(r, i)$, where Latin letters denote boundary components. Plugged in the total action Eq.(4), this evaluation produces

$$
\begin{aligned}
I_{0}= & \frac{1}{16 \pi G(D-3)(D-4)} \int_{\mathcal{M}} d^{D-1} x d r \sqrt{-h} N \delta_{\left[i_{1} \cdots i_{4}\right]}^{\left[j_{1} \cdots j_{4}\right]}\left[\left(\frac{1}{2} R_{j_{1} j_{2}}^{i_{1} i_{2}}+\frac{1}{\ell^{2}} \delta_{j_{1}}^{i_{1}} \delta_{j_{2}}^{i_{2}}+\frac{2}{D-2} R_{r i_{1}}^{r i_{1}} \delta_{j_{2}}^{i_{2}}\right) \delta_{j_{3}}^{i_{3}} \delta_{j_{4}}^{i_{4}}\right. \\
& \left.+\alpha(D-3)\left(\frac{D-4}{4} R_{j_{1} j_{2}}^{i_{1} i_{2}}+2 R_{r j_{1}}^{r i_{1}} \delta_{j_{2}}^{i_{2}}\right) R_{j_{3} j_{4}}^{i_{3} i_{4}}\right]+\int_{\mathcal{M}} d^{D-1} x d r \sqrt{-h} N \mathcal{L}\left(F^{2}\right),
\end{aligned}
$$

where we have used the fact that $\delta_{\left[r i_{1} \cdots i_{4}\right]}^{\left[r j_{1} \cdots j_{4}\right]}=\delta_{\left[i_{1} \cdots i_{4}\right]}^{\left[j_{1} \cdots j_{4}\right]}$. One can recognize the component $\mathcal{E}_{r}^{r}$ of the equations of motion in the form (A5) (see Appendix A) from the first two terms in the first line and the first term in the second one. In doing so, the action in radial normal frame reads

$$
\begin{gathered}
I_{0}=\frac{1}{8 \pi G(D-2)(D-3)} \int_{\mathcal{M}} d^{D-1} x d r \sqrt{-h} N \delta_{\left[i_{1} \cdots i_{3}\right]}^{\left[j_{1} \cdots j_{3}\right]} R_{r i_{1}}^{r i_{1}}\left(\delta_{j_{2}}^{i_{2}} \delta_{j_{3}}^{i_{3}}+\alpha(D-2)(D-3) R_{j_{2} j_{3}}^{i_{2} i_{3}}\right) \\
-\int_{\mathcal{M}} d^{D-1} x d r \sqrt{-h} N\left(\frac{1}{8 \pi G} \mathcal{E}_{r}^{r}+T_{r}^{r}-\mathcal{L}\left(F^{2}\right)\right) .
\end{gathered}
$$

We consider a spacetime given by the Euclidean continuation of the static black hole metric (14), where the Euclidean time $\tau=i t$ appears as identified in a period $\beta$, which is the inverse of the Hawking temperature

$$
T \equiv \beta^{-1}=\left.\frac{1}{4 \pi} \frac{d f^{2}(r)}{d r}\right|_{r=r_{+}}
$$

As the horizon is reduced to a single point, the Euclidean period in Eq.(32) smooths out the origin of the radial coordinate $r=r_{+}$, avoiding the presence of a cone-like singularity. We stress the fact that no boundary is introduced at the horizon, such that no extra surface terms are needed there in order to reproduce the correct black hole thermodynamics. 
More explicitly, the Hawking temperature is given by

$$
T=\frac{1}{4 \pi r_{+}} \frac{(D-3) k+\frac{(D-1) r_{+}^{2}}{\ell^{2}}+\alpha(D-3)(D-4)(D-5) \frac{k^{2}}{r_{+}^{2}}+\frac{16 \pi G r_{+}^{2}}{D-2} T_{r}^{r}\left(q, r_{+}\right)}{1+2 \alpha(D-3)(D-4) \frac{k}{r_{+}^{2}}}
$$

where the electromagnetic field contributes to the black hole temperature trough the tensor $T_{r}^{r}\left(q, r_{+}\right)=\mathcal{L}+4 E^{2} \frac{d \mathcal{L}}{d F^{2}}$ evaluated at $F^{2}=-2 E^{2}$ and for $r=r_{+}$.

The Wick rotation implies $I^{E}=-i I$ for the Euclidean action, that can be expressed in terms of the black hole metric function $f^{2}$ and its derivatives as

$$
\begin{aligned}
I_{0}^{E}= & \frac{1}{16 \pi G} \int_{0}^{\beta} d \tau \int_{\Gamma_{D-2}} \sqrt{\gamma} d^{D-2} \varphi \int_{r_{+}}^{\infty} d r r^{D-2}\left[\left(f^{2}\right)^{\prime \prime}+(D-2) \frac{\left(f^{2}\right)^{\prime}}{r}+\right. \\
& \left.+\frac{2 \alpha(D-2)(D-3)}{r^{2}}\left(\left(f^{2}\right)^{\prime \prime}\left(k-f^{2}\right)-\left(f^{2}\right)^{\prime 2}+(D-4) \frac{\left(f^{2}\right)^{\prime}\left(k-f^{2}\right)}{r}\right)\right]- \\
& +\left.4 \int_{0}^{\beta} d \tau \int_{\Gamma_{D-2}} \sqrt{\gamma} d^{D-2} \varphi \int_{r_{+}}^{\infty} d r r^{D-2} E^{2} \frac{d \mathcal{L}}{d F^{2}}\right|_{F^{2}=-2 E^{2}},
\end{aligned}
$$

where we have substituted the expressions for the Riemann tensor in Appendix $\mathbb{C}$ and the electromagnetic stress tensor (19). Upon a trivial integration on the boundary coordinates, all the integrand can be written as a total derivative, and the bulk Euclidean action becomes

$$
\begin{aligned}
I_{0}^{E}= & \left.\frac{\beta \operatorname{Vol}\left(\Gamma_{D-2}\right)}{16 \pi G}\left[\left(f^{2}\right)^{\prime}\left(r^{D-2}+2 \alpha(D-2)(D-3) r^{D-4}\left(k-f^{2}\right)\right)\right]\right|_{r_{+}} ^{\infty} \\
& -\left.\beta \operatorname{Vol}\left(\Gamma_{D-2}\right)\left(4 r^{D-2} \phi E \frac{d \mathcal{L}}{d F^{2}}\right)\right|_{r_{+}} ^{\infty},
\end{aligned}
$$

where, in the last line, the equation of motion for NED field (20) was used.

By definition of the Euclidean period $\beta$, the first line evaluated at the horizon $r_{+}$produces $-S$, where $S$ is the standard value of black hole entropy in EGB gravity [29, 30]

$$
S=\frac{\operatorname{Vol}\left(\Gamma_{D-2}\right) r_{+}^{D-2}}{4 G}\left(1+\frac{2 k \alpha}{r_{+}^{2}}(D-2)(D-3)\right)
$$

whereas the second line is $\beta Q \Phi$, where

$$
Q=4 \operatorname{Vol}\left(\Gamma_{D-2}\right) q
$$

Notice that, what is physically relevant for the thermodynamic description of NED action, is the potential difference between the horizon and infinity. Therefore, we assume $\phi(\infty)=0$ without loss of generality.

Thus, the Euclidean continuation of the bulk action for EGB AdS gravity coupled to NED is written as

$$
I_{0}^{E}=\beta Q \Phi-S+\frac{\beta \operatorname{Vol}\left(\Gamma_{D-2}\right)}{16 \pi G} \lim _{r \rightarrow \infty}\left[\left(f^{2}\right)^{\prime}\left(r^{D-2}+2 \alpha(D-2)(D-3) r^{D-4}\left(k-f^{2}\right)\right)\right],
$$


what, in terms of the black hole mass [18, 31,33

$$
M=(D-2) \frac{\operatorname{Vol}\left(\Gamma_{D-2}\right) \mu}{16 \pi G},
$$

can be recast as

$$
\begin{aligned}
I_{0}^{E}= & \beta Q \Phi-S+\beta M \frac{D-2}{D-3} \frac{\ell_{e f f}^{2}-2 \alpha(D-2)(D-5)}{\ell_{e f f}^{2}-2 \alpha(D-3)(D-4)}+ \\
& +\frac{\beta \operatorname{Vol}\left(\Gamma_{D-2}\right)}{16 \pi G} \lim _{r \rightarrow \infty}\left[\frac{r^{D-3}}{\ell_{e f f}^{2}}\left(1+2 \alpha(D-2)(D-3) r^{D-4}\left(k-f^{2}\right)\right)\right] .
\end{aligned}
$$

The unusual factor multiplying $\beta M$ indicates that a background-subtraction method would not necessarily give rise to a correct QSR. Indeed, subtracting the value of $I_{0}^{E}$ evaluated for AdS vacuum (with the corresponding AdS radius $\ell_{\text {eff }}$ ) gets rid of the divergences at $r=\infty$, but does not reproduce the mass in the asymptotic region. The quantity that appears at radial infinity is the analog of Komar formula for EGB gravity [34].

It is possible to understand the above result in the light of Iyer-Wald definition of conserved quantities in gravity theories 35]. This time-honored procedure interprets the black hole entropy as the Noether charge $\tilde{Q}[\xi]$ for a Killing vector $\xi=\xi^{\mu} \partial_{\mu}$, evaluated at the horizon. This quantity, however, is derived exclusively from the bulk Lagrangian of the gravity theory (which in our case is denoted by $\mathcal{L}_{\text {grav }}$ ), without additional boundary terms. More concretely, the charge $\tilde{Q}[\xi]$ is written in terms of the derivative of the bulk Lagrangian respect to the Lorentz curvature two-form. In tensorial notation, we can work out an alternative form if the Lagrangian density is re-written as $\mathcal{L}_{\text {grav }}=\delta_{\left[\nu_{1} \cdots \nu_{D}\right]}^{\left[\mu_{1} \cdots \mu_{D}\right]} L_{\mu_{1} \cdots \mu_{D}}^{\nu_{1} \cdots \nu_{D}}$. In this way, the charge is given by the fully-covariant formula in terms of derivatives of the Riemann tensor

$$
\tilde{Q}[\xi]=\int_{\partial \mathcal{M}_{r} \cap \Xi_{t}} d^{D-2} x \sqrt{-g} \hat{n}_{\mu} \hat{u}_{\nu} \xi^{\lambda} \delta_{\left[\nu_{1} \cdots \nu_{D}\right]}^{\left[\mu_{1} \cdots \mu_{D}\right]} g^{\alpha \sigma} \Gamma_{\lambda \sigma}^{\beta} \frac{\delta L_{\mu_{1} \cdots \mu_{D}}^{\nu_{1} \cdots \nu_{D}}}{\delta R_{\mu \nu}^{\alpha \beta}},
$$

which must be evaluated at the intersection of a $r=$ const. boundary $\partial \mathcal{M}_{r}$ with a constant-time slice $\Xi_{t}\left[35\right.$ 37]. The normal vectors $\hat{n}_{\mu}$ and $\hat{u}_{\mu}$ generate the corresponding foliations which describe $\partial \mathcal{M}_{r}$ and $\Xi_{t}$, respectively 38 ].

The form of the action (29) for EGB gravity is particularly useful to compute the above expression, what produces

$$
\begin{aligned}
\tilde{Q}[\xi]=-\frac{1}{16} & \frac{1 G(D-2)(D-3)}{\mathcal{M}_{r} \cap \Xi_{t}} d^{D-2} x \sqrt{-g} \hat{n}_{\mu_{1}} \hat{u}_{\mu_{2}} \xi^{\lambda} \delta_{\left[\nu_{1} \nu_{2} \nu_{3} \nu_{4}\right]}^{\left[\mu_{1} \mu_{2} \mu_{3} \mu_{4}\right]} g^{\nu_{1} \alpha} \Gamma_{\lambda \alpha}^{\nu_{2}} \times \\
& \times\left(\delta_{\left[\mu_{3} \mu_{4}\right]}^{\left[\nu_{3} \nu_{4}\right]}+2(D-2)(D-3) \alpha R_{\mu_{3} \mu_{4}}^{\nu_{3} \nu_{4}}\right) .
\end{aligned}
$$

In the Gauss-normal frame (28), the normal derivative of the induced metric $h_{i j}$ describes the extrinsic properties of the boundary,

$$
K_{i j}=-\frac{1}{2 N} h_{i j}^{\prime}
$$


what defines the extrinsic curvature. Then the Christoffel symbol can be expressed in terms of $K_{i j}$ (see Appendix B), such that the Iyer-Wald charge is

$$
\begin{aligned}
& \tilde{Q}[\xi]=\frac{1}{16 \pi G(D-2)(D-3)} \int_{\partial \mathcal{M}_{r} \cap \Xi_{t}} d^{D-2} x \sqrt{-h} \hat{u}_{j} \xi^{i} \delta_{\left[i_{1} \cdots i_{D-1}\right]}^{\left[j j_{2} \cdots j_{D-1}\right]} K_{i}^{i_{1}} \times \\
& \times\left(\delta_{\left[j_{2} j_{3}\right]}^{\left[i_{2} i_{3}\right]}+2 \alpha(D-2)(D-3) R_{j_{2} j_{3}}^{i_{2} i_{3}}\right),
\end{aligned}
$$

where $\sqrt{-g}=N \sqrt{-h}$. It is not difficult to show that, for the black hole metric (14), the Noether charge (44) leads to

$$
\tilde{Q}\left[\partial_{t}\right]=\frac{\operatorname{Vol}\left(\Gamma_{D-2}\right)}{16 \pi G}\left(f^{2}\right)^{\prime}\left(r^{D-2}+2 \alpha(D-2)(D-3) r^{D-4}\left(k-f^{2}\right)\right),
$$

for an arbitrary surface $\Gamma_{D-2}$ of radius $r$. This quantity clearly reproduces the value of the entropy (36) when evaluated at the horizon $r=r_{+}$, and a finite contribution (which cannot be identified with the mass) plus a divergent term at infinity, which are the same terms present in Eq.(40). The fact that $I_{0}^{E}$ can be written down as

$$
I_{0}^{E}=\beta Q \Phi+\left.\beta \tilde{Q}\left[\partial_{t}\right]\right|_{r_{+}} ^{\infty},
$$

means that the problem of finiteness of the Euclidean action is connected to the regularization of the Noether charges in the asymptotic region.

It is possible to correct the Iyer-Wald formula, such that it provides the correct mass and angular momentum for black holes, through the construction of a Hamiltonian $H[\xi]$ which describes the dynamics generated by the vector field $\xi^{\mu}$. Generally speaking, when one varies the action respect to the fields $\phi$, one identifies the equations of motion plus a surface term

$$
\delta I=\int_{\mathcal{M}}(E O M) \delta \phi+\int_{\partial \mathcal{M}} \Theta(\phi, \delta \phi) .
$$

Then, the Hamiltonian is related to the Iyer-Wald charge at radial infinity $\tilde{Q}_{\infty}[\xi]=\int_{\Sigma_{\infty}} \tilde{\mathcal{Q}}(\xi)$ and surface term in eq.(47) by

$$
\delta H[\xi]=\delta \int_{\Sigma_{\infty}} \tilde{\mathcal{Q}}(\xi)-\int_{\Sigma_{\infty}} \xi \cdot \Theta(\phi, \delta \phi),
$$

where $\Sigma_{\infty}=\partial \mathcal{M} \cap \Xi_{t}$. The Hamiltonian exists if there is a $(D-1)$-form $\mathcal{B}$ such that the second term is a total variation,

$$
\int_{\Sigma_{\infty}} \xi \cdot \Theta(\phi, \delta \phi)=\delta \int_{\Sigma_{\infty}} \xi \cdot \mathcal{B}(\phi) .
$$

Whenever this is possible, one can write down the Wald Hamiltonian as 35]

$$
H[\xi]=\int_{\Sigma_{\infty}}(\tilde{\mathcal{Q}}(\xi)-\xi \cdot \mathcal{B}) .
$$


However, the procedure outlined above in general breaks covariance at the boundary. As a result, the correction $\mathcal{B}$ to the charge has a non-covariant form, and has to be built on a case-bycase basis for different solutions. Moreover, if exists, $\mathcal{B}$ cannot be used as an appropriate boundary term to render the Euclidean action finite.

In what follows, we employ covariant counterterms given as polynomials in the extrinsic and intrinsic curvatures to regularize the Euclidean action and to obtain the QSR given by Eq.(11).

\section{KOUNTERTERM REGULARIZATION AND QUANTUM STATISTICAL RELATION}

The regularization of gravitational action prescribed by AdS/CFT correspondence leads to the addition of covariant functionals of the boundary metric and curvature, known as counterterm series. In a dual quantum field theory, this counterterm series corresponds to a standard UV divergence removal by adding finite polynomials in the fields.

However, in EGB-AdS gravity, holographic renormalization becomes too involved to provide a general answer to this problem.

Here, we use an alternative regularization procedure, where the counterterms depend explicitly on the extrinsic curvature. The choice of such terms in AdS gravity is justified by the asymptotic expansions of the fields. Indeed, the extrinsic curvature and the boundary metric are proportional at the leading order. In that way, the boundary term $B_{D-1}$ is given as a unique geometrical structure depending only on the dimension. This approach circumvents the technicalities of holographic methods for higher-curvature theories of the Lovelock class [39].

We will work with gravity-NED action in $D$ dimensions supplemented by a boundary term

$$
I=I_{0}+c_{D-1} \int_{\partial \mathcal{M}} d^{D-1} x B_{D-1},
$$

where the coupling $c_{D-1}$ is fixed demanding a well-defined action principle [18, 28]. The explicit form of the Kounterterm series as a polynomial of the extrinsic and intrinsic curvatures for $\mathrm{EH}$ AdS gravity was introduced in Refs. [40, 41].

For the current discussion, we consider the grand canonical ensemble, where the temperature $T$ and the electric potential $\Phi$ are held fixed at the horizon. The Gibbs free energy

$$
G(T, \Phi)=U-T S+\Phi Q
$$

that satisfies the differential equation

$$
d G=-S d T+Q d \Phi,
$$

is given in terms of the Euclidean action as

$$
G=T I^{E} .
$$

When the partition function in semiclassical approximation is written in terms of Gibbs energy as

$$
Z=e^{-G / T} .
$$


In what follows, we employ Kounterterms to regulate the value of the Euclidean action for EGB-AdS coupled to NED and to obtain the QSR (1) for charged black hole solutions.

\section{A. Even dimensions}

In a manifold $\mathcal{M}$ without boundary in even dimensions $D=2 n$, the integration of the Euler topological invariant produces the Euler characteristic, $\chi(\mathcal{M})$. If a boundary $\partial \mathcal{M}$ is introduced, there appears a correction to $\chi(\mathcal{M})$ given by the $n$-th Chern form

$$
B_{2 n-1}=2 n \sqrt{-h} \int_{0}^{1} d t \delta_{\left[i_{1} \cdots i_{2 n-1}\right]}^{\left[j_{1} \cdots j_{2 n-1}\right]} K_{j_{1}}^{i_{1}}\left(\frac{1}{2} \mathcal{R}_{j_{2} j_{3}}^{i_{2} i_{3}}-t^{2} K_{j_{2}}^{i_{2}} K_{j_{3}}^{i_{3}}\right) \cdots\left(\frac{1}{2} \mathcal{R}_{j_{2 n-2} j_{2 n-1}}^{i_{2 n-2} i_{2 n-1}}-t^{2} K_{j_{2 n-2}}^{i_{2 n-2}} K_{j_{2 n-1}}^{i_{2 n-1}}\right) .
$$

Here, $\mathcal{R}_{k l}^{i j}(h)$ is the intrinsic curvature of the boundary, related to the spacetime Riemann tensor by $R_{k l}^{i j}=\mathcal{R}_{k l}^{i j}-K_{k}^{i} K_{l}^{j}+K_{l}^{i} K_{k}^{j}$ (see Appendix (B). In asymptotically AdS spacetimes, the addition of the above boundary term to the gravity action defines an extrinsic regularization scheme in even dimensions. In EGB AdS gravity, it has been shown in Ref. [18] that the constant $c_{2 n-1}$ in front of the boundary term $B_{2 n-1}$ has to be fixed in terms of the effective AdS radius as

$$
c_{2 n-1}=-\frac{1}{16 \pi G} \frac{\left(-\ell_{e f f}^{2}\right)^{n-1}}{n(2 n-2) !}\left(1-\frac{2 \alpha}{\ell_{\text {eff }}^{2}}(2 n-2)(2 n-3)\right),
$$

in order to cancel the divergences in the Euclidean action. Notice that the integration on the continuous parameter $t$ generates the coefficients if one wants to express the boundary term as a polynomial. This compact way of writing $B_{2 n-1}$ is not a mere formality but it reflects the relation to topological invariants and provides a useful tool for explicit evaluations, as well.

The boundary term (56) evaluated on the Euclidean continuation of the black hole metric (14) becomes

$$
\begin{aligned}
\int_{\partial \mathcal{M}} d^{2 n-1} x B_{2 n-1}^{E}= & -2 n \lim _{r \rightarrow \infty} \int_{0}^{\beta} d \tau \int_{\Gamma_{D-2}} \sqrt{\gamma} d^{D-2} \varphi r^{2 n-2} f \int_{0}^{1} d t \delta_{\left[n_{1} \cdots n_{2 n-2}\right]}^{\left[m_{1} \cdots m_{2 n-2}\right]} \\
& \times K_{\tau}^{\tau}\left(\frac{1}{2} \mathcal{R}_{m_{1} m_{2}}^{n_{1} n_{2}}-(2 n-1) t^{2} K_{m_{1}}^{n_{1}} K_{m_{2}}^{n_{2}}\right) \times \\
& \left(\frac{1}{2} \mathcal{R}_{m_{3} m_{4}}^{n_{3} n_{4}}-t^{2} K_{m_{3}}^{n_{3}} K_{m_{4}}^{n_{4}}\right) \cdots\left(\frac{1}{2} \mathcal{R}_{m_{2 n-3} m_{2 n-2}}^{n_{2 n-3} n_{2 n-2}}-t^{2} K_{m_{2 n-3}}^{n_{2 n-3}} K_{m_{2 n-2}}^{n_{2 n-2}}\right) .
\end{aligned}
$$

Substituting the components of the extrinsic curvature (C1) and intrinsic curvature (C2) (see Appendix (C) and performing the integral

$$
\int_{0}^{1} d t\left[k-(2 n-1) t^{2} f^{2}\right]\left(k-t^{2} f^{2}\right)^{n-2}=\left(k-f^{2}\right)^{n-1},
$$

the boundary term takes the form

$c_{2 n-1} \int_{\partial \mathcal{M}} d^{2 n-1} x B_{2 n-1}^{E}=\left.\frac{\beta \operatorname{Vol}\left(\Gamma_{D-2}\right) \ell_{e f f}^{2 n-2}}{16 \pi G}\left(1-\frac{2 \alpha}{\ell_{e f f}^{2}}(2 n-2)(2 n-3)\right)\left(f^{2}\right)^{\prime}\left(f^{2}-k\right)^{n-1}\right|^{r=\infty}$. 
In consequence, the total Euclidean action

$$
I_{2 n}^{E}=I_{0}^{E}+c_{2 n-1} \int_{\partial \mathcal{M}} d^{2 n-1} x B_{2 n-1}^{E}
$$

can be written as

$$
\begin{aligned}
I_{2 n}^{E}= & \frac{\beta \operatorname{Vol}\left(\Gamma_{D-2}\right)}{16 \pi G}\left\{\left.\left(f^{2}\right)^{\prime}\left(r^{D-2}+2 \alpha(2 n-2)(2 n-3) r^{D-4}\left(k-f^{2}\right)\right)\right|_{r_{+}} ^{\infty}\right. \\
& \left.-\left.\ell_{\text {eff }}^{2 n-2}\left(1-\frac{2 \alpha}{\ell_{\text {eff }}^{2}}(2 n-2)(2 n-3)\right)\left[\left(f^{2}\right)^{\prime}\left(f^{2}-k\right)^{n-1}\right]\right|^{r=\infty}\right\}+\beta Q \Phi .
\end{aligned}
$$

The contribution at infinity from the bulk action combines with the one from the boundary to produce $\beta$ times the Noether mass $M$

$$
\begin{aligned}
M & =\frac{\operatorname{Vol}\left(\Gamma_{2 n-2}\right)}{16 \pi G} \lim _{r \rightarrow \infty} r^{2 n-2}\left(f^{2}\right)^{\prime}\left[1-2 \alpha(2 n-2)(2 n-3) \frac{f^{2}-k}{r^{2}}-\right. \\
& \left.-\left(1-\frac{2 \alpha(2 n-2)(2 n-3)}{\ell_{e f f}^{2}}\right) \ell_{e f f}^{2 n-2}\left(\frac{f^{2}-k}{r^{2}}\right)^{n-1}\right],
\end{aligned}
$$

which agrees with the formula (39) when expressed in terms of $\mu$ [28]. In other words, because Kounterterm series leads to the cancellation of divergences in the asymptotic charges, the finiteness of the Euclidean action is ensured for any static black hole and satisfies the QSR

$$
G(T, \Phi)=T I_{2 n}^{E}=U-T S+Q \Phi
$$

where the EGB black hole entropy is given by Eq.(36).

\section{B. Odd dimensions}

In $D=2 n+1$ dimensions, Kounterterm regularization provides the explicit form of the boundary terms which makes the Euclidean action finite in EH AdS gravity [40]. The universality of the Kounterterm series ensures that the action will be also finite in any Lovelock gravity with AdS branches. That means that the information on a particular theory is incorporated in the regularization scheme through effective AdS radius $\ell_{\text {eff }}$ and the coupling constant $c_{2 n}$. Thus, in general, the Kounterterms series is given in terms of the parametric integrations

$$
B_{2 n}=\frac{n}{2^{n-2}} \sqrt{-h} \int_{0}^{1} d t \int_{0}^{t} d s \delta_{\left[i_{1} \cdots i_{2 n}\right]}^{\left[j_{1} \cdots j_{2 n}\right]} K_{j_{1}}^{i_{1}} \delta_{j_{2}}^{i_{2}} \mathcal{F}_{j_{3} j_{4}}^{i_{3} i_{4}}(t, s) \cdots \mathcal{F}_{j_{2 n-1} j_{2 n}}^{i_{2 n-1} i_{2 n}}(t, s),
$$

where we introduce an auxiliary quantity with the symmetries of the Riemann tensor

$$
\mathcal{F}_{k l}^{i j}(t, s)=\mathcal{R}_{k l}^{i j}-t^{2}\left(K_{k}^{i} K_{l}^{j}-K_{l}^{i} K_{k}^{j}\right)+\frac{s^{2}}{\ell_{e f f}^{2}} \delta_{[k l]}^{[i j]},
$$


such that in EGB AdS theory the coupling reads [18]

$$
\begin{aligned}
c_{2 n} & =-\frac{1}{16 \pi G} \frac{\left(-\ell_{e f f}^{2}\right)^{n-1}}{n(2 n-1) !}\left(1-\frac{2 \alpha(2 n-1)(2 n-2)}{\ell_{e f f}^{2}}\right)\left[\int_{0}^{1} d t\left(1-t^{2}\right)^{n-1}\right]^{-1} \\
& =-\frac{1}{16 \pi G} \frac{2\left(-\ell_{e f f}^{2}\right)^{n-1}}{n(2 n-1) ! \beta\left(n, \frac{1}{2}\right)}\left(1-\frac{2 \alpha(2 n-1)(2 n-2)}{\ell_{e f f}^{2}}\right),
\end{aligned}
$$

where $\beta\left(n, \frac{1}{2}\right)=\frac{2^{2 n-1}(n-1) !^{2}}{(2 n-1) !}$ is the Beta function for those arguments.

In the Euclidean sector, the boundary term in the black hole ansatz (14) produces

$$
\begin{aligned}
\int_{\partial \mathcal{M}} d^{2 n} x B_{2 n}^{E}= & -\frac{n}{2^{n-3}} \lim _{r \rightarrow \infty} \int_{0}^{\beta} d \tau \int_{\Gamma_{D-2}} \sqrt{\gamma} d^{D-2} \varphi r^{2 n-1} f \int_{0}^{1} d t \int_{0}^{t} d s \delta_{\left[n_{1} \cdots n_{2 n-1}\right]}^{\left[m_{1} \cdots m_{2 n-1}\right]} \mathcal{F}_{m_{4} m_{5} n_{5}}^{n_{1}} \cdots \mathcal{F}_{m_{2 n-2} m_{2 n-1}}^{n_{2 n-2} n_{2 n-1}} \\
& \times\left[\left(K_{\tau}^{\tau} \delta_{m_{1}}^{n_{1}}+K_{m_{1}}^{n_{1}}\right) \mathcal{F}_{m_{2} m_{3}}^{n_{2} n_{3}}+2(n-1) K_{m_{1}}^{n_{1}} \delta_{m_{2}}^{n_{2}}\left(-t^{2} K_{\tau}^{\tau} K_{m_{3}}^{n_{3}}+\frac{s^{2}}{\ell_{e f f}^{2}} \delta_{m_{3}}^{n_{3}}\right)\right]
\end{aligned}
$$

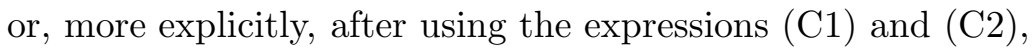

$$
\begin{aligned}
\int_{\partial \mathcal{M}} d^{2 n} x B_{2 n}^{E}= & 2 n(2 n-1) ! \beta \operatorname{Vol}\left(\Gamma_{2 n-1}\right) \lim _{r \rightarrow \infty} \int_{0}^{1} d t \int_{0}^{t} d s\left(k-t^{2} f^{2}+s^{2} \frac{r^{2}}{\ell_{e f f}^{2}}\right)^{n-2} \times \\
& {\left[\frac{r\left(f^{2}\right)^{\prime}}{2}\left(k-(2 n-1) t^{2} f^{2}+s^{2} \frac{r^{2}}{\ell_{e f f}^{2}}\right)+f^{2}\left(k-t^{2} f^{2}+(2 n-1) s^{2} \frac{r^{2}}{\ell_{e f f}^{2}}\right)\right] }
\end{aligned}
$$

In order to recognize the asymptotic charges from the Euclidean action, it is especially convenient to convert the above double integrals into a single-parameter integration, i.e.,

$$
\begin{aligned}
& \int_{0}^{1} d t \int_{0}^{t} d s\left(k-(2 n-1) t^{2} f^{2}+s^{2} \frac{r^{2}}{\ell_{e f f}^{2}}\right)\left(k-t^{2} f^{2}+s^{2} \frac{r^{2}}{\ell_{e f f}^{2}}\right)^{n-2} \\
= & \int_{0}^{1} d t\left(k-f^{2}+t^{2} \frac{r^{2}}{\ell_{e f f}^{2}}\right)^{n-1}-\int_{0}^{1} d t t\left(k-t^{2} f^{2}+t^{2} \frac{r^{2}}{\ell_{e f f}^{2}}\right)^{n-1}
\end{aligned}
$$

and

$$
\int_{0}^{1} d t \int_{0}^{t} d s\left(k-t^{2} f^{2}+(2 n-1) s^{2} \frac{r^{2}}{\ell_{e f f}^{2}}\right)\left(k-t^{2} f^{2}+s^{2} \frac{r^{2}}{\ell_{e f f}^{2}}\right)^{n-2}=\int_{0}^{1} d t t\left(k-t^{2} f^{2}+t^{2} \frac{r^{2}}{\ell_{e f f}^{2}}\right)^{n-1} .
$$

Thus, the surface term is expressed as

$$
\begin{aligned}
\int_{\partial \mathcal{M}} d^{2 n} x B_{2 n}^{E}=n(2 n-1) ! & \beta \operatorname{Vol}\left(\Gamma_{2 n-1}\right) \lim _{r \rightarrow \infty}\left[r^{2 n-1}\left(f^{2}\right)^{\prime} \int_{0}^{1} d t\left(\frac{k-f^{2}}{r^{2}}+\frac{t^{2}}{\ell_{e f f}^{2}}\right)^{n-1}+\right. \\
+ & \left.2\left(f^{2}-\frac{r\left(f^{2}\right)^{\prime}}{2}\right) \int_{0}^{1} d t t\left(k-t^{2} f^{2}+t^{2} \frac{r^{2}}{\ell_{e f f}^{2}}\right)^{n-1}\right] .
\end{aligned}
$$


When the above boundary term is added to the bulk Euclidean action (35) with a suitable coupling constant,

$$
I_{2 n+1}^{E}=I_{0}^{E}+c_{2 n} \int_{\partial \mathcal{M}} d^{2 n} x B_{2 n}^{E}
$$

one gets

$$
\begin{aligned}
I_{2 n+1}^{E}= & \left.\frac{\beta \operatorname{Vol}\left(\Gamma_{2 n-1}\right)}{16 \pi G}\left[r^{2 n-1}\left(f^{2}\right)^{\prime}\left(1-2 \alpha(D-2)(D-3) \frac{f^{2}-k}{r^{2}}\right)\right]\right|_{r_{+}} ^{\infty} \\
& +\beta \operatorname{Vol}\left(\Gamma_{2 n-1}\right) n c_{2 n}(2 n-1) !\left[r^{2 n-1}\left(f^{2}\right)^{\prime} \int_{0}^{1} d t\left(\frac{k-f^{2}}{r^{2}}+\frac{t^{2}}{\ell_{\text {eff }}^{2}}\right)^{n-1}\right. \\
& \left.+2 \int_{0}^{1} d t t\left(f^{2}-\frac{r\left(f^{2}\right)^{\prime}}{2}\right)\left(k+t^{2}\left(\frac{r^{2}}{\ell_{\text {eff }}^{2}}-f^{2}\right)\right)^{n-1}\right]\left.\right|^{\infty}+\beta Q \Phi .
\end{aligned}
$$

Thus, the contribution coming from radial infinity in first two lines can be identified with $\beta M$, where the mass is [28]

$$
\begin{aligned}
M & =\frac{\operatorname{Vol}\left(\Gamma_{2 n-1}\right)}{16 \pi G} \lim _{r \rightarrow \infty} r^{2 n-1}\left(f^{2}\right)^{\prime}\left[1+2 \alpha(2 n-1)(2 n-2) \frac{k-f^{2}}{r^{2}}+\right. \\
& \left.+16 \pi G(2 n-1) ! n c_{2 n} \int_{0}^{1} d t\left(\frac{k-f^{2}}{r^{2}}+\frac{t^{2}}{\ell_{e f f}^{2}}\right)^{n-1}\right],
\end{aligned}
$$

which agrees with the mass formula (39) when the metric function is expanded. The term with parametric integration in the third line of Eq.(72) is $\beta$ times the vacuum energy $E_{v a c}$, which is written in the form

$$
E_{v a c}=2 n(2 n-1) ! c_{2 n} \operatorname{Vol}\left(\Gamma_{2 n-1}\right) \lim _{r \rightarrow \infty} \int_{0}^{1} d t t\left(f^{2}-\frac{r\left(f^{2}\right)^{\prime}}{2}\right)\left[k+\left(\frac{r^{2}}{\ell_{e f f}^{2}}-f^{2}\right) t^{2}\right]^{n-1} .
$$

The consistency of the black hole thermodynamics is therefore verified through the QSR, which involves the black hole entropy (36)

$$
G(T, \Phi)=T I_{2 n+1}^{E}=U-T S+Q \Phi
$$

for a total energy which includes the zero-point energy $E_{v a c}$,

$$
U=M+E_{v a c} .
$$

\section{Thermodynamic charges}

Until now, we have obtained the QSR (11) for electrically charged black holes in gravitating NED, which involves the total energy $U$ and the electric charge $Q$ as asymptotic Noether charges, computed in Ref.[28], and the entropy $S$ as the Wald charge at the horizon. However, strictly 
speaking, one should be able to reproduce these quantities from thermodynamic relations in an independent way.

From that point of view, the gravitational entropy should be calculated from the Gibbs free energy as

$$
S_{T D}=-\left(\frac{\partial G}{\partial T}\right)_{\Phi}
$$

and the electric charge

$$
Q_{T D}=\left(\frac{\partial G}{\partial \Phi}\right)_{T}
$$

whereas the internal energy can be derived as a thermodynamic quantity from

$$
U_{T D}=G-T\left(\frac{\partial G}{\partial T}\right)_{\Phi}-\Phi\left(\frac{\partial G}{\partial \Phi}\right)_{T} .
$$

In general, arbitrary variations of $G$ in Eq.(52) produce in terms of the Noether charges

$$
d G=d U-T d S+\Phi d Q-S d T+Q d \Phi .
$$

It is clear that if Noether charges satisfy the First Law (2), then the relations (77) 79 ) identify thermodynamic with Noether charges.

In order to prove the First Law, it is convenient to introduce the variable $\eta$,

$$
\eta \equiv \frac{q}{r_{+}^{D-2}}
$$

and write the electric charge as

$$
Q\left(r_{+}, \eta\right)=4 \operatorname{Vol}\left(\Gamma_{D-2}\right) \eta r_{+}^{D-2} .
$$

A more explicit expression for the Hawking temperature is

$$
T\left(r_{+}, \eta\right)=\frac{(D-3) k+\frac{(D-1) r_{+}^{2}}{\ell^{2}}+\alpha(D-3)(D-4)(D-5) \frac{k^{2}}{r_{+}^{2}}+\frac{16 \pi G r_{+}^{2}}{D-2}\left(\mathcal{L}_{+}-4 \eta E_{+}\right)}{4 \pi r_{+}\left(1+2 \alpha(D-3)(D-4) \frac{k}{r_{+}^{2}}\right)}
$$

where the index ' + ' denotes quantities evaluated at $r=r_{+}$.

The Generalized Gauss law,

$$
\left.E_{+} \frac{\partial \mathcal{L}}{\partial F^{2}}\right|_{r_{+}}=-\eta
$$

determines the electric field $E_{+}=E\left(r_{+}, q\right)$ such that it is a function of $\eta$ only, and so are $\mathcal{L}_{+}$and its first derivative. A useful relation is

$$
\frac{\partial \mathcal{L}_{+}}{\partial \eta}=\left.\frac{\partial \mathcal{L}}{\partial F^{2}}\right|_{r_{+}} \frac{\partial F^{2}}{\partial \eta}=4 \eta \frac{\partial E_{+}}{\partial \eta} .
$$


From the definition of horizon, $f\left(r_{+}\right)=0$, we also obtain

$$
U\left(r_{+}, \eta\right)=E_{v a c}+(D-2) \frac{\operatorname{Vol}\left(\Gamma_{D-2}\right)}{16 \pi G}\left[k r_{+}^{D-3}+k^{2} \alpha(D-3)(D-4) r_{+}^{D-5}+\frac{r_{+}^{D-1}}{\ell^{2}}+\frac{16 \pi G \mathcal{T}_{+}}{D-2}\right],
$$

where $E_{v a c}$ is vanishing for even dimensions and $\mathcal{T}_{+}$is the function (24) evaluated at the horizon,

$$
\begin{aligned}
\mathcal{T}_{+} & =\left.\frac{1}{D-1}\left(r^{D-1} \mathcal{L}-4 q r E+(D-2) 4 q \phi\right)\right|_{\infty} ^{r_{+}} \\
& =\frac{1}{D-1}\left(r_{+}^{D-1} \mathcal{L}_{+}-4 r_{+}^{D-1} \eta E_{+}-(D-2) 4 r_{+}^{D-2} \eta \Phi\right) .
\end{aligned}
$$

Finally, the electric potential between infinity and the event horizon has the form

$$
\Phi\left(r_{+}, \eta\right)=-\frac{r_{+} \eta^{\frac{1}{D-2}}}{D-2} \int_{0}^{\eta} d u u^{-\frac{D-1}{D-2}} E\left(r_{+}, u\right)
$$

where $u=q / r^{D-2}=\eta\left(r_{+} / r\right)^{D-2}$.

When varied, the entropy, charge and total energy change as

$$
\begin{aligned}
& d S= \frac{\operatorname{Vol}\left(\Gamma_{D-2}\right)}{4 G}(D-2) r_{+}^{D-3}\left(1+2 \alpha(D-3)(D-4) \frac{k}{r_{+}^{2}}\right) d r_{+}, \\
& d Q= 4 \operatorname{Vol}\left(\Gamma_{D-2}\right) r_{+}^{D-3}\left((D-2) \eta d r_{+}+r_{+} d \eta\right), \\
& d U=(D-2) \frac{\operatorname{Vol}\left(\Gamma_{D-2}\right)}{16 \pi G} r_{+}^{D-4}\left[(D-3) k+\alpha(D-3)(D-4)(D-5) \frac{k^{2}}{r_{+}^{2}}\right. \\
&\left.\quad+(D-1) \frac{r_{+}^{2}}{\ell^{2}}\right] d r_{+}+\operatorname{Vol}\left(\Gamma_{D-2}\right) d \mathcal{T}_{+} .
\end{aligned}
$$

The last line involves the variation of $\mathcal{T}_{+}$which, using Eq.(87) and the variation of $\Phi$,

$$
d \Phi=\frac{\Phi}{r_{+}} d r_{+}+\frac{\Phi-r_{+} E_{+}}{(D-2) \eta} d \eta,
$$

can be left in the more suitable form

$$
d \mathcal{T}_{+}=\left[r_{+}^{D-2}\left(\mathcal{L}_{+}-4 \eta E_{+}\right)-4(D-2) r_{+}^{D-3} \eta \Phi\right] d r_{+}-4 r_{+}^{D-2} \Phi d \eta
$$

With the formula for $T$ given by Eq.83), we finally find that

$$
\begin{aligned}
d U= & T(D-2) \frac{\operatorname{Vol}\left(\Gamma_{D-2}\right)}{4 G} r_{+}^{D-3}\left(1+2 \alpha(D-3)(D-4) \frac{k}{r_{+}^{2}}\right) d r_{+} \\
& -4 \operatorname{Vol}\left(\Gamma_{D-2}\right) r_{+}^{D-3}\left((D-2) \eta d r_{+}+r_{+} d \eta\right) \Phi
\end{aligned}
$$

from where we recognize the variations $d S$ and $d Q$ as in Eqs. (89) and (90), such that the First Law holds. 
In this case, the Gibbs free energy satisfies Eq.(53) and, as a consequence, the thermodynamic relations (77 79) are also valid for the corresponding Noether charges, what implies

$$
\begin{aligned}
S_{T D} & =S, \\
Q_{T D} & =Q, \\
U_{T D} & =U=M+E_{v a c} .
\end{aligned}
$$

It is worthwhile stressing that, while the proof of First Law is insensitive to the presence of the vacuum energy $E_{v a c}$, the last equation defines the internal energy as the total energy of the black hole, as expected in the context of AdS/CFT correspondence.

\section{CONCLUSIONS}

In this paper, we have studied the thermodynamics of topological static AdS black holes in Einstein-Gauss-Bonnet theory in the presence of an electric field described by an arbitrary nonlinear electrodynamics. This is done using a background-independent regularization scheme which considers the addition of counterterms depending on both the intrinsic and extrinsic curvature of the boundary metric, to the action.

The fact that the Kounterterm series is given as a compact expression, i.e., in a parametric representation of the polynomials, provides us with a practical tool to evaluate the regularized action in all dimensions. Likewise, this also makes easier recognizing the quantities appearing at radial infinity as the conserved quantities of the theory. This issue is particularly relevant, because it connects the finiteness of the Euclidean action with the problem of regularization of the asymptotic charges treated in Ref.[28] and, ultimately, with a well-posed variational principle.

In the standard counterterms method, it is not possible to separate the contributions to the quasilocal stress tensor that produce the black hole mass $M$ from those which give rise to the vacuum energy. This means that in the evaluation of the Euclidean action $I^{E}$ one cannot clearly identified the term $\beta E_{v a c}$ from the terms at $r=\infty$ in all odd dimensions. Nonetheless, one could work out a relation between the Kounterterms and the intrinsic prescription given by holographic renormalization, by taking the asymptotic expansion of the extrinsic curvature (for a similar procedure in Einstein-Hilbert gravity, see Ref.[42]).

We also performed an independent checking, which is obtaining the total energy $U$ and electric charge $Q$ as thermodynamic quantities from Eqs.(79) and (78), respectively. As expected, the internal energy contains a Casimir contribution in odd dimensions, consisting with the Noether approach.

The generality of the procedure is a consequence of the fact that, in the context of Kounterterm regularization, the Quantum Statistical Relation appears as a thermodynamic identity of the gravity-NED action plus boundary terms. This suggests an extension of the above results to other Lovelock theories and, probably, to other matter couplings, as long as one can define the asymptotic behavior in terms of the curvature as in Eq.(13) (for a thermodynamic study of higher derivative corrections in the Abelian gauge field of the form $F^{4}$, see Ref.[43]). 


\section{Acknowledgments}

This work was funded by FONDECYT Grants 11070146, 1090357 and 1100755. O.M. is supported in part by Project MECESUP UCV0602 and the PUCV through the projects 123.797/2007 and $123.705 / 2010$.

\section{Appendix A: Useful identities}

The totally-antisymmetric Kronecker delta of rank $p$ is defined as the determinant

$$
\delta_{\left[\mu_{1} \cdots \mu_{p}\right]}^{\left[\nu_{1} \cdots \nu_{p}\right]}:=\left|\begin{array}{cccc}
\delta_{\mu_{1}}^{\nu_{1}} & \delta_{\mu_{1}}^{\nu_{2}} & \cdots & \delta_{\mu_{1}}^{\nu_{p}} \\
\delta_{\mu_{2}}^{\nu_{1}} & \delta_{\mu_{2}}^{\nu_{2}} & & \delta_{\mu_{2}}^{\nu_{p}} \\
\vdots & & \ddots & \\
\delta_{\mu_{p}}^{\nu_{1}} & \delta_{\mu_{p}}^{\nu_{2}} & \cdots & \delta_{\mu_{p}}^{\nu_{p}}
\end{array}\right|
$$

A contraction of $k \leq p$ indices in the Kronecker delta of rank $p$ produces a delta of rank $p-k$,

$$
\delta_{\left[\mu_{1} \cdots \mu_{k} \cdots \mu_{p}\right]}^{\left[\nu_{1} \cdots \nu_{k} \cdots \nu_{p}\right]} \delta_{\nu_{1}}^{\mu_{1}} \cdots \delta_{\nu_{k}}^{\mu_{k}}=\frac{(N-p+k) !}{(N-p) !} \delta_{\left[\mu_{k+1} \cdots \mu_{p}\right]}^{\left[\nu_{k+1} \cdots \nu_{p}\right]}
$$

where $N$ is the range of indices.

Using this compact notation, the Einstein tensor (9) can be rewritten in terms of the AdS radius as

$$
G_{\nu}^{\mu}=-\frac{1}{2} \delta_{\left[\nu \nu_{1} \nu_{2}\right]}^{\left[\mu \mu_{1} \mu_{2}\right]}\left(\frac{1}{2} R_{\mu_{1} \mu_{2}}^{\nu_{1} \nu_{2}}+\frac{1}{\ell^{2}} \delta_{\mu_{1}}^{\nu_{1}} \delta_{\mu_{2}}^{\nu_{2}}\right)
$$

and, in a similar fashion, the Laczos tensor (10) adopts the form

$$
H_{\nu}^{\mu}=-\frac{\alpha}{8} \delta_{\left[\nu \nu_{1} \cdots \nu_{4}\right]}^{\left[\mu \mu_{1} \cdots \mu_{4}\right]} R_{\mu_{1} \mu_{2}}^{\nu_{1} \nu_{2}} R_{\mu_{3} \mu_{4}}^{\nu_{3} \nu_{4}}
$$

In order to identify the equation of motion for the metric in the evaluation of the Euclidean action, it is convenient to employ the above relations to convert (17) into

$$
\begin{aligned}
\mathcal{E}_{\nu}^{\mu}=-\frac{1}{2(D-3)(D-4)} \delta_{\left[\nu \nu_{1} \cdots \nu_{4}\right]}^{\left[\mu \mu_{1} \cdots \mu_{4}\right]}\left[\frac{1}{2} R_{\mu_{1} \mu_{2}}^{\nu_{1} \nu_{2}} \delta_{\mu_{3}}^{\nu_{3}} \delta_{\mu_{4}}^{\nu_{4}}+\right. \\
\left.\quad+\frac{1}{\ell^{2}} \delta_{\mu_{1}}^{\nu_{1}} \delta_{\mu_{2}}^{\nu_{2}} \delta_{\mu_{3}}^{\nu_{3}} \delta_{\mu_{4}}^{\nu_{4}}+\frac{\alpha}{4}(D-3)(D-4) R_{\mu_{1} \mu_{2}}^{\nu_{1} \nu_{2}} R_{\mu_{3} \mu_{4}}^{\nu_{3} \nu_{4}}\right]-8 \pi G T_{\nu}^{\mu} .
\end{aligned}
$$

\section{Appendix B: Gauss-normal coordinate frame}

In the Gauss-normal coordinate system (28), the only relevant components of the connection $\Gamma_{\mu \nu}^{\alpha}$ are expressed in terms of the extrinsic curvature $K_{i j}=-\frac{1}{2 N} h_{i j}^{\prime}$ as

$$
\Gamma_{i j}^{r}=\frac{1}{N} K_{i j}, \quad \Gamma_{r j}^{i}=-N K_{j}^{i}, \quad \Gamma_{r r}^{r}=\frac{N^{\prime}}{N} .
$$


The radial foliation (28) implies the Gauss-Codazzi relations for the spacetime curvature, as well,

$$
\begin{aligned}
R_{k l}^{i r} & =\frac{1}{N}\left(\nabla_{l} K_{k}^{i}-\nabla_{k} K_{l}^{i}\right), \\
R_{k r}^{i r} & =\frac{1}{N}\left(K_{k}^{i}\right)^{\prime}-K_{l}^{i} K_{k}^{l}, \\
R_{k l}^{i j} & =\mathcal{R}_{k l}^{i j}(h)-K_{k}^{i} K_{l}^{j}+K_{l}^{i} K_{k}^{j},
\end{aligned}
$$

where $\nabla_{i}=\nabla_{i}(h)$ is the covariant derivative defined in the Christoffel symbol of the boundary $\Gamma_{i j}^{k}(g)=\Gamma_{i j}^{k}(h)$ and $\mathcal{R}_{k l}^{i j}(h)$ is the intrinsic curvature of the boundary.

\section{Appendix C: Topological black hole metric}

In the static topological black hole ansatz (14), the extrinsic curvature takes the form

$$
K_{j}^{i}=-\frac{1}{2 N} h^{i k} h_{k j}^{\prime}=\left(\begin{array}{cc}
-f^{\prime} & 0 \\
0 & -\frac{f}{r} \delta_{n}^{m}
\end{array}\right),
$$

where prime denotes radial derivative, and the non-vanishing components of the boundary curvature are

$$
\mathcal{R}_{m_{1} m_{2}}^{n_{1} n_{2}}(h)=\frac{k}{r^{2}} \delta_{\left[m_{1} m_{2}\right]}^{\left[n_{1} n_{2}\right]}
$$

The spacetime Riemann tensor $R_{\lambda \rho}^{\mu \nu}$ is then given by

$$
\begin{aligned}
R_{t r}^{t r} & =-\frac{1}{2}\left(f^{2}\right)^{\prime \prime}, \\
R_{t m}^{t n} & =R_{r m}^{r n}=-\frac{1}{2 r}\left(f^{2}\right)^{\prime} \delta_{m}^{n}, \\
R_{k l}^{m n} & =\frac{1}{r^{2}}\left(k-f^{2}\right) \delta_{[k l]}^{[m n]} .
\end{aligned}
$$

[1] J.M. Maldacena, The large $N$ limit of superconformal field theories, Adv. Theor. Math. Phys. 2, 231 (1998); Int. J. Theor. Phys. 38, 1113 (1999). hep-th/9711200]; S.S. Gubser, I.R. Klebanov and A.M. Polyakov, A semiclassical limit of the gauge string correspondence, Nucl. Phys. B636, 99 (2002). hep-th/0204051; E. Witten, Anti-de Sitter space and holography, Adv. Theor. Math. Phys. 2, 253 (1998). hep-th/9802150.

[2] P. Kovtun, D. T. Son and A. O. Starinets, Holography and hydrodynamics: diffusion on stretched horizons, J. High Energy Phys. 10, 064 (2003). hep-th/0309213

[3] M. Brigante, H. Liu, R. C. Myers, S. Shenker and S. Yaida, Viscosity bound violation in higher derivative gravity, Phys. Rev. D77: 126006 (2008). arXiv:0712.0805; Y. Kats and P. Petrov, Effect of curvature squared corrections in AdS on the viscosity of the dual gauge theory, J. High Energy Phys. 01: 044 (2009). arXiv:0712.0743.

[4] R-G. Cai and Y-W. Sun, Shear viscosity from AdS Born-Infeld black holes, J. High Energy Phys. 09: 115 (2008). arXiv:0807.2377. 
[5] X.H. Ge, Y. Matsuo, F.W. Shu, S.J. Sin and T. Tsukioka, Viscosity bound, causality violation and instability with stringy correction and charge, J. High Energy Phys. 10, 009 (2008). arXiv:0808.2354.

[6] R. Gregory, S. Kanno and J. Soda, Holographic superconductors with higher curvature corrections, J. High Energy Phys. 10: 010 (2009). arXiv:0907.3203

[7] Q. Y. Pan, B. Wang, E. Papantonopoulos, J. Oliveira and A. Pavan, Holographic superconductors with various condensates in Einstein-Gauss-Bonnet gravity, Phys. Rev. D81: 106007 (2010). arXiv:0912.2475

[8] J. Jing and S. Chen, Holographic superconductors in the Born-Infeld electrodynamics, Phys. Lett. B686: 68 (2010). arXiv:1001.4227; J. Jing, L. Wang, Q. Pan and S. Chen, Holographic superconductors in Gauss-Bonnet gravity with Born-Infeld electrodynamics. arXiv:1012.0644]

[9] G.W. Gibbons, M.J. Perry and C.N. Pope, The First law of thermodynamics for Kerr-anti-de Sitter black holes, Class. Quant. Grav. 22, 1503 (2005). hep-th/0408217

[10] Following the arguments given in Ref.[9], we adopt this nomenclature for this thermodynamic formula.

[11] This fact is a consequence of breaking $S O(2 n, 2)$ invariance by the action of radial diffeomorphisms.

[12] V. Balasubramanian and P. Kraus, A stress tensor for anti-de Sitter gravity, Commun. Math. Phys. 208, 413 (1999). hep-th/9902121]

[13] R. Emparan, C.V. Johnson and R.C. Myers, Surface terms as counterterms in the AdS/CFT correspondence, Phys.Rev. D 60, 104001 (1999). hep-th/9903238; R.B. Mann, Misner string entropy, Phys. Rev. D 60, 1040047 (1999). hep-th/9903229

[14] M. Henningson and K. Skenderis, The holographic Weyl anomaly, J. High Energy Phys. 07, 023 (1998). hep-th/9806087

[15] S. de Haro, K. Skenderis and S. Solodukhin, Holographic reconstruction of space-time and renormalization in the AdS/CFT correspondence, Commun. Math. Phys. 217, 595 (2001). hep-th/0002230

[16] Y. Brihaye and E. Radu, Black objects in the Einstein-Gauss-Bonnet theory with negative cosmological constant and the boundary counterterm method, J. High Energy Phys. 09, 006 (2008). arXiv:0806.1396.

[17] J.T. Liu and W.A. Sabra, Hamilton-Jacobi counterterms for Einstein-Gauss-Bonnet gravity, Class. Quant. Grav. 27, 175014 (2010). arXiv:0807.1256.

[18] G. Kofinas and R. Olea, Vacuum energy in Einstein-Gauss-Bonnet AdS gravity, Phys. Rev. D74, 084035 (2006). hep-th/0606253

[19] O. Miskovic and R. Olea, Thermodynamics of Einstein-Born-Infeld black holes with negative cosmological constant, Phys. Rev. D77, 124048 (2008). arXiv:0802.2081.

[20] W. Heisenberg and H. Euler, Folgerungen aus der Diracschen Theorie des Positrons, Z. Phys. 98, 714 (1936). Translation by W. Korolevski and H. Kleinert, Consequences of Dirac's Theory of the Positron. physics/0605038

[21] M. Born and I. Infeld, Foundations of the new field theory, Proc. R. Soc. A144, 425 (1934).

[22] E.S. Fradkin and A.A. Tseytlin, Nonlinear electrodynamics from quantized strings, Phys. Lett. B163, 123 (1985).

[23] B. Hoffmann, Gravitational and electromagnetic mass in the Born-Infeld electrodynamics, Phys. Rev. 47, 877 (1935); G. W. Gibbons and D. A. Rasheed, Electric-magnetic duality rotations in nonlinear electrodynamics, Nucl. Phys. B454, 185 (1995).

[24] H.P. de Oliveira, Nonlinear charged black holes, Class. Quant. Grav. 11, 1469 (1994).

[25] H.H. Soleng, Charged black points in general relativity coupled to the logarithmic U(1) gauge theory, Phys. Rev. D52, 6178 (1995). hep-th/9509033.

[26] H. Maeda, M. Hassaine and C. Martinez, Lovelock black holes with a nonlinear Maxwell field, Phys. Rev. D79, 044012 (2009). arXiv:0812.2038

[27] D. Boulware and S. Deser, String generated gravity models, Phys. Rev. Lett. 55, 2656 (1985). 
[28] O. Miskovic and R. Olea, Conserved charges for black holes in Einstein-Gauss-Bonnet gravity coupled to nonlinear electrodynamics in AdS space, Phys. Rev. D83: 024011 (2011). arXiv:1009.5763.

[29] R-G. Cai, Gauss-Bonnet black holes in AdS spaces, Phys. Rev. D65: 084014 (2002). hep-th/0109133

[30] M. Cvetic, S. Nojiri and S.D. Odintsov, Black hole thermodynamics and negative entropy in de Sitter and anti-de Sitter Einstein-Gauss-Bonnet gravity Nucl. Phys. B628, 295 (2002). hep-th/0112045

[31] S. Deser and B. Tekin, Energy in generic higher curvature gravity theories, Phys. Rev. D67: 084009 (2003). hep-th/0212292

[32] A. Padilla, Surface terms and the Gauss-Bonnet Hamiltonian, Class. Quant. Grav. 20: 3129 (2003). gr-qc/0303082

[33] E. Gravanis, Conserved charges in (Lovelock) gravity in first order formalism, Phys. Rev. D81: 084013 (2010). arXiv:1004.3582,

[34] For a discussion on Komar formula for Lovelock gravity theories see, e.g., D. Kastor, Komar integrals in higher (and lower) derivative gravity, Class. Quant. Grav. 25: 175007 (2008). arXiv:0804.1832.

[35] V. Iyer and R.M. Wald, Some properties of Noether charge and a proposal for dynamical black hole entropy, Phys. Rev. D50, 846 (1994). gr-qc/9403028

[36] T. Clunan, S.F. Ross and D.J. Smith, On Gauss-Bonnet black hole entropy, Class. Quant. Grav. 21, 3447 (2004). gr-qc/0402044

[37] A. Paranjape, S. Sarkar and T. Padmanabhan, Thermodynamic route to field equations in LancosLovelock gravity, Phys. Rev. D74: 104015 (2006). hep-th/0607240)

[38] The reader might be confused with the unusual dependence on the Christoffel symbol of Eq. (41) as compared with the formulas appearing in the literature. However, it is straightforward to prove that the charge can be equivalently written in terms of the covariant derivative of the vector $\xi^{\mu}$.

[39] G. Kofinas and R. Olea, Universal regularization prescription for Lovelock AdS gravity, J. High Energy Phys. 11, 069 (2007). [arXiv: 0708.0782]; Universal Kounterterms in Lovelock AdS gravity, Fortsch. Phys. 56, 957 (2008). arXiv:0806.1197.

[40] R. Olea, Regularization of odd-dimensional AdS gravity: Kounterterms, J. High Energy Phys. 04, 073 (2007). hep-th/0610230

[41] R. Olea, Mass, angular momentum and thermodynamics in four-dimensional Kerr-AdS black holes, J. High Energy Phys. 06, 023 (2005). hep-th/0504233

[42] O. Miskovic and R. Olea, Topological regularization and self-duality in four-dimensional anti-de Sitter gravity, Phys. Rev. D79, 124020 (2009). arXiv:0902.2082

[43] D. Anninos and G. Pastras, Thermodynamics of the Maxwell-Gauss-Bonnet anti-de Sitter black hole with higher derivative gauge corrections, J. High Energy Phys. 07, 030 (2009). arXiv:0807.3478, 